\section{Decompositions of a set into disjoint pairs}

\section{S. Ginsburg (University of Miami)}

In this note we shall discuss the possibility of the decomposition of a set of elements into disjoint pairs such that every set which is the union of pairs and which satisfies one specified property, also satisfies a second specified property. The principal result, Theorem 1.1, is a generalization of a lemma due to Sierpiński [3].

1. THEOREM 1.1. Let $F=\left\{A_{\xi} \mid \xi<\tau\right\}$ be a family of abstract sets, each set being of power $\kappa_{\gamma}$. Furthermore, let $F$ contain a coinitial subfamily $G=\left\{B_{\xi} \mid \xi<\omega_{\gamma}\right\}$, i. e., for each $\xi<\tau, A_{\xi}$ contains as a subset, at least one element of $G$. Then there exists a decomposition of $A=\cup A_{\xi}$ into disjoint pairs of elements with the following property: For any set $S$ which is the union of pairs, if $S$ contains at least one set $A_{\xi}$, then $S$ has $\kappa_{\gamma}$ elements in common with each element of $F$.

Proof. The demonstration is a modification of the proof of Lemma 1 of [3].

In Theorem 1 of [2] Sierpiński stated a general result on families of sets. An inspection of the proof reveals that the following result was proved at the same time:

(*) If $\left\{D_{\xi}\right\}_{\xi<\omega_{\gamma}}$ is a sequence of sets, each set being of power $\kappa_{\gamma}$, then there exists a family $P=\left\{N_{\xi} \mid \xi<\omega_{\gamma}\right\}$ of pairwise disjoint sets, each set of power $<\kappa_{\gamma}$, such that $1^{0} \bigcup_{\xi<\omega_{\gamma}} N_{\xi}=\bigcup_{\xi<\omega_{\gamma}} D_{\xi}$, and $2^{0}$ any set $Q$ which is the union of a family $T$ of $\kappa_{\gamma}$, sets in $P$ has at least one element in. common with each $D_{\xi}$.

Since $T$ is the union of $\kappa_{\gamma}$ disjoint families of $\kappa_{\xi}$ setis each and since the $N_{\xi}$ are pairwise disjoint, it follows that $Q$ has $\kappa_{\gamma}$ elements in common with each $D_{\xi}$.

Turning to the proof of Theorem 1.1 let $M$ be the family of those sets $B$ in $G$ for which the power of $A-B$ is s. Let $H=M \cup\{A-B \mid B \in M\}$.

A. Suppose that $H$ is non-empty. Well order the elements of $H$ into a sequence $\left\{D_{\xi}\right\}_{\xi<\omega_{\gamma}}$ where the $D_{\xi}$ are not necessarily all distinct. Then $\cup D_{\xi}=A$. Let $P \stackrel{\omega_{\nu}}{=}\left\{N_{\xi} \mid \xi<\omega_{\gamma}\right\}$ be the family of sets obtained by applying $(*)$ to our sequence $\left\{D_{\xi}\right\}_{\xi}<\omega_{\gamma}$. Decompose the set of ordinal numbers $T=\left\{\xi \mid \xi<\omega_{\gamma}\right\}$ into a family $\left\{W_{\xi} \mid \xi<\omega_{\gamma}\right\}$ of pairwise disjoint sets, each set being of power $s_{\gamma}$. For $\delta<\omega_{\gamma}$ let $C_{\delta}=\cup N_{v}$. Thus $\cup C_{\delta}=A$, and for $\xi, \delta<\omega_{\gamma}, D_{\xi} \cap C_{\delta}$ is of power $\kappa_{\gamma}$. From the definition of $H$, for each $\xi, \mathcal{A}-D_{\xi}$ is in $H$. Therefore the power of the set $C_{\xi} \cap\left(A-D_{\xi}\right)$ is $\mathrm{s}_{\gamma}$. Denote by $\left\{x_{v j v<\omega_{\gamma}}\right.$ and by $\left\{y_{n}^{\xi}\right\}_{v<\omega_{y}}$, the elements of $C_{\xi} \cap D_{\xi}$ and $C_{\xi} \cap\left(A-D_{\xi}\right)$ respectively. Thus

$$
\left.\bigcup_{n,<\omega_{\gamma}}\left(x_{v}^{\xi}, y_{v}^{\xi}\right)=\left(D_{\xi} \cap\left(C_{\xi}\right) \cup[) A-D_{\xi}\right) \cap C_{\xi}\right]=A \cap O_{\xi}=C_{\xi} .
$$

Consider the set of pairs $K=\left\{\left(x_{v}^{\xi}, y_{v}^{\xi}\right) \mid \xi<\omega_{y}, v<\omega_{y}\right\}$. Since $A$ is the union of the pairwise disjoint sets $C_{\xi}$, it follows that $A$ is the union of the pairwise disjoint pairs in $K$.

Now let $S$ be any set which is the union of pairs and which contains an element of $F$. By hypothesis, there exists an element $B$ in $G$ for which $B C S$.

(i) Suppose that $B$ is in $G-M$. For each $\xi, D_{\xi}=\left(D_{\xi} \cap B\right) \cup\left[D_{\xi} \cap(A-B)\right]$. Since $D_{\xi}$ is of power s, and $A-B$ is of power $<\kappa_{y}, D_{\xi} \cap B$ is of power $s_{y}$. Since $G$ is coinitial in $F$, it follows that $B \cap A_{\varepsilon}$ is of power $s_{\gamma}$ for each $\xi<\tau$. Thus $S \cap A_{\xi}$ is of power s, for $\xi<\tau$.

(ii) Suppose that $B$ is in $I I$, say $B=D_{\xi}$. Therefore $C_{\xi} \cap D_{\xi} \subseteq S$. Let $x$ be any element of $\left(A-D_{\xi}\right) \cap C_{\xi}$. From the definition of the pairs there exists an element $y$ in $D_{\xi} \cap C_{\xi}$ such that $(x, y)$ is a pair. Since $S$ is the union of pairwise disjoint pairs, and $x$ is in $S$, it follows that $y$ is also in $S$. Consequently $\left(A-D_{\xi}\right) \cap C_{\xi} \subseteq S$. Hence

$$
\left[\left(A-D_{\xi}\right) \cup C_{\xi}\right] \cup\left(D_{\xi} \cap C_{\xi}\right)=C_{\xi} \subseteq S .
$$

For each $v<\omega_{\gamma}, C_{\xi} \cap D_{v}$, therefore $S \cap D_{v}$, is of power $s_{\gamma}$. For an element $D$ of $G-M I$, we have seen in (i) that $D \cap D_{\xi}$, thus $D \cap S$, is of power $s_{\gamma}$. Thus $D_{\xi} \cap B_{v}$, for $v<\omega_{\gamma}$, is of power $s_{\gamma}$. Therefore $D_{\xi} \cap A_{\sigma}$, for $\sigma<\tau$, is of power $s_{y}$.

B. Suppose that $M$ is empty, $i$. e., there is no set $B_{\xi}$ sueh that $A-B_{\xi}$ is of power $s_{\gamma}$. Decompose $A$, in an arbitrary manner, into disjoint pairs. Using (i) above it is easily seen that this decomposition satisfies the conclusion of the theorem, q. e. d.

COROLLARY. Let $K$ be an infinite set of power $s_{y}$. The family $A$ of all subsets of $K$, of power $\mathrm{s}_{y}$ each, can be decomposed into disjoint pairs with the following property: If $S$ is any subfamily of $A$, consisting of pairs, and if $S$ contains some element $Y$ of $A$ together with all subsets of $Y$ in $A$, then for each element $Z$ in $A, S$ contains an element $U$ in $A$ which is a subset of $Z, i$. e., $S$ is a coinitial subfamily of the family $A$, ordered by set inclusion. 
Remarks. 1. Assume that the hypothesis of Theorem 1.1 is fulfilled. For a given decomposition of $A$ into disjoint pairs it is possible that each set $S$, of power $s_{\gamma}$, which is the union of pairs, has an intersection with each set in $F$ of power s. For example, let $A$ be an abstract set of power $s_{\gamma}, A=\left\{x_{\xi}, y_{\xi} \mid \xi<\omega_{\gamma}\right\}$. For each $\xi$ let $\left(x_{\xi}, y_{\xi}\right)$ be a pair and let

$$
A_{\xi}=\left\{x_{v} \mid v<\omega_{\gamma}\right\} \cup\left\{y_{\xi}\right\} \quad \text { and } \quad B_{\xi}=\left\{y_{v} \mid v \neq \xi\right\} \text {. }
$$

Denote by $F(=G)$ the family of sets $\left\{A_{\xi}, B_{\xi} \mid \xi<\omega_{\gamma}\right\}$. The hypothesis of Theorem 1.1 is satisfied. Now each set $\$$, of power $s_{\gamma}$, which consists of pairs, contains $\kappa_{\gamma}$ of the $x$ 's and $y$ 's. For each $\xi<\omega_{\gamma}, A_{\xi}$ and $B_{\xi}$ contains all but a finite number of the $x$ 's and the $y$ 's respectively. Consequently each of the sets, $A_{\xi} \cap S$ and $B_{\xi} \cap S$, is of power $\kappa_{\gamma}$.

The situation is different if the family $F$ contains at least three disjoint sets, say $A_{0}, A_{1}$, and $A_{2}$. For any decomposition of $A$ into disjoint pairs, there always exists a set $\delta$, of power $s_{\gamma}$, consisting of pairs, which has an empty intersection with some set in $F$, in particular, with either $A_{1}$ or $A_{2}$. To see this let $Z$ be the set of those elements which are in pairs that are in $A_{0}$. Thus $Z$ is a subset of $A_{0}$. If the power of $Z$ is $s_{\gamma}$, let $S=Z$. Since $A_{0}$ and $A_{1}$ are disjoint, $S$ and $A_{1}$ are disjoint. If the power of $Z$ is $\left\langle\boldsymbol{s}_{\gamma}\right.$, then the set $U$, defined as the set $A_{0}-Z$, is of porrer $\boldsymbol{s}_{\gamma}$ This is so because each set in $F$ is of power $s_{\gamma}$. For each element $u$ in $U$, let $(u, v)$ be the pair which contains $u$, and let $V=\{v \mid v \in(u, v), u \in U\}$ If $s_{\gamma}$ of the elements of $V$ are in $A_{1}$ let $S$ be the union of those pairs $(u, v)$, where $u$ is in $U$ and $v$ is in $A_{1}$. Since $A_{0} A_{1}$ and $A_{2}$ are pairwise disjoint, $S \cap A_{2}$ is empty. If there are fewrer than $s_{\gamma}$ elements of $V$ in $A_{1}$ let $S$ be the union of those pairs $(u, v)$, where $u$ is in $U$ and $v$ is not in $A_{1}$. Here $S \cap A_{1}$ is empty. It is clear that in each case, the power of $S$ is $\kappa_{\gamma}$.

2. From remark 1 and Theorem 1.1 we see that if $F$ contains three disjoint sets, then there exists a set $S$, of power $s_{\gamma}$, consisting of pairs, which contains no set in $F$. The conclusion of the previous statement holds even if $F$ does not contain three disjoint sets. To be specific the following result will now be proved:

Let a set $A$, of power $s_{\gamma}$, be decomposed into disjoint pairs. Iror any family of sets $F=\left\{A_{\xi} \mid \xi<\omega_{\gamma}\right\}$, of power $\kappa_{\gamma}$ each, there exists a set $\mathcal{S}$, of power $s_{y}$, consisting of pairs, which contains as a subset no set in. $F$. Fruthermore, for each $\xi, S \cap A_{\xi}$ is non-empty.

- In order to see this let the elements of $A$ and the pairs in $A$ each be well ordered into the two sequences, $\left\{p_{\xi}\right\}_{\xi<\omega_{\gamma}}$ and $\left\{a_{\xi}\right\}_{\xi<\omega_{\gamma}}$ respectively. Let $x_{0}$ be the first element in $A_{0}$ and let $b_{0}$ be the pair containing $x_{0}$ Let $y_{0}$ be the first element in the set $A_{0}-b_{0}$ and $c_{0}$ the pair containing $y_{0}$ We continue by transfinite induction. For each $\xi<a<\omega_{\gamma}$ let $x_{\xi}, y_{\xi}, b_{\xi}$, and $c_{\xi}$ be defined. Let $x_{a}$ be the first element in the set $A_{a}-\left[\bigcup_{\xi<a}\left(b_{\xi} \cup c_{\xi}\right)\right]$ and let $b_{\alpha}$ be the pair which contains $x_{\alpha}$. Let $y_{\alpha}$ be the first element in the set $A_{a}-\left[\bigcup_{\xi<\alpha}\left(b_{\xi} \cup c_{\xi}\right) \cup b_{a}\right]$ and let $c_{a}$ be the pair which contains $y_{\alpha}$. Clearly the elements $x_{\xi}, x_{v}, y_{\sigma}, y_{\tau}, \xi \neq v, \sigma \neq \tau$ are in different pairs. Now define $s$ as the set $L b_{\xi}$. For each $\xi, y_{\xi}$ is an element of $A_{\xi}$ which Now define $s$ as the sew is not in $S$. Consequently $S$ contains no set in $F$.

2. We now restrict ourselves to decompositions of the plane. We denote the plane by $E^{2}$.

THEOREM 2.1. There is no decomposition of the plane into disjoint pairs with the following property: S being any set which is the union of pairs, if $s$ contains one line then it contains tuo lines.

Proof. Suppose the contrary, i. e., suppose that such a decomposition does exist. For each line $L$ denote by $S(L)$ the union of the pairs $(x, y)$, where $x$ is in $L$. Let $L_{1}$ be any line. By assumption, $S\left(L_{1}\right)$ contains a second line, say $L_{2}$. Consider the set $S\left(L_{2}\right)$. $S\left(L_{2}\right)$ consists of the union of pairs, each pair containing an element in $L_{2}$ and an element in $L_{1}$. Suppose that $L_{1}$ contains a pair neither of whose elements is in $L_{2}$. Then $S\left(I_{2}\right)$ cannot contain $L_{1}$. Thus $S\left(L_{2}\right)$ contains but one line. From this contradiction we obtain the following:

(*) For each line $L_{1}$ there corresponds a second line $L_{2}$ so that $S\left(L_{1}\right)=S\left(L_{2}\right)$. Cnder this correspondance $L_{1}$ corresponds to $L_{2}$. To each element $x$ in $L_{1}$ there corresponds an element $y$ in $L_{2}$ such that, for $x \neq y,(x, y)$ is a pair. If $L_{1} \cap L_{2}$ is non-empty, say $L_{1} \cap L_{2}=\left\{x_{0}\right\}$, then the pair $\left(x_{0}, y_{0}\right)$ which contains $x_{0}$, need not be a subset of $L_{1} \cup L_{2}, i$.e., $y_{0}$ need not be in either $L_{1}$ or $L_{2}$.

Now let $(p, q)$ be any pair and let $L_{1}$ be the line containing $p$ and $q$. Let $L_{2}$ be the line which corresponds to $L_{1}$. By $(*), L_{2}$ contains either $p$ or $q$, say $p$. Let $(r, s) \neq(p, q)$ be any pair with $r$ in $L_{1}$ and $s$ in $L_{2}$. Denote bo $I_{3}$ the line which contains $q$ and $s$. Clearly $L_{3}$ is neither $L_{1}$ nor $L_{2}$. Denote by $L_{4}$ the line corresponding to $L_{3}$. By $(*), L_{4}$ is neither $L_{1}$ nor $L_{2}$. Now the elements $q$ and $s$ belong to $S\left(L_{3}\right)$. From (*), if $L_{4}$ does not contain $p$, then $L_{4}$ contains $q$ and $r$. Since $L_{1}$ contains $q$ and $r, L_{4}$ is $L_{1}$. If $L_{4}$ does not contain $q$, then $L_{4}$ contains $p$. Now $L_{4}$ contains either $r$ or $s$. If $L_{4}$ contains $r$, then $L_{4}$ is $L_{1}$. If $L_{4}$ contains $s$, then $L_{4}$ is $L_{2}$. In any case $L_{4}$ is either $L_{1}$ or $L_{2}$. This is a contradiction. Hence no such decomposition is possible, q.e.d.

If we only demand a decomposition such that each $S(L)$ contain a line segment not on $L$, then such a decomposition can be effected. To be precise we have 
THEOREM 2.2. There exists a decomposition of the plane into disjoint pairs with the following property: Every set which is the union of pairs and which contains one line

$1^{0}$ contains $2^{*_{0}}$ disjoint, non-trivial closed intervals of lines in the plane,

$2^{0}$ has $2^{\mathrm{x}_{0}}$ elements in common with every line in the plane.

Proof. Denote by $K$ the set of points in the plane

$$
K=\{(x, y) \mid-\infty<x<\infty, 0 \leqslant y \leqslant 1\} .
$$

For each line $L$ which is not entirely in $K$, let $L^{\prime}=L-K$. Let $\theta$ be the smallest ordinal number whose power is $2^{\mathrm{s}_{0}}$. Let $F^{\prime}=Q^{\prime}$ $=\left\{B_{\xi} \mid \xi<\theta\right\}$ be the family of all such sets $L^{\prime}$. Obviously each set $L^{\prime}$ contains $2^{\mathrm{s}_{0}}$ elements. On applying the same procedure tio the sequence $\left\{B_{\xi}\right\}_{\xi<0}$ as in the proof of Theorem 1.1 we obtain a family of pairwise disjoint sets $\left\{C_{\xi}^{\prime} \mid \xi<\theta\right\}$ whose union is $E^{2}-K$, and such that $C_{\xi}^{\prime} \cap B_{n}$, is of powrer $2^{*_{0}}$ for all $\xi, v<\theta$. For each real number $x$ define $D_{x}$ as the set $D_{x}=\{(x, y) \mid 0 \leqslant y \leqslant 1\}$. Since the power of the real numbers is $2^{s_{0}}$, we may decompose the set of real numbers into a family $\left\{E_{\xi} \mid \xi<\theta\right\}$ of pairwise disjoint sets, each set being of power $2^{*_{0}}$. For each $\xi$ let $C_{\xi}=C_{\xi}^{\prime} \cup \bigcup_{x \in E_{\xi}} D_{x}$. Note that each set $C_{\xi}$ has $2^{\aleph_{0}}$ elements in common with each line in the plane. Let $F(=G)$ be the set of all lines in the plane. Repeating the proof of Theorem 1.1 on $F$ and the $C_{\xi}$, we obtain a decomposition of $E^{2}$ into disjoint pairs. Any set $S$ which is the union of pairs and which contains a line, contains some set $C_{\xi}$. Therefore $S$ satisfies the conclusions of the theorem.

Finally we have

THEOREM 2.3. There exists a decomposition of the plane into a family of aisjoint pairs with the following property: For each line $L$ in the plane, the set $S(L)$, which is the union of the pairs each of which is entively in $L$, is exact ${ }^{1}$ ) and has property $\left.A^{2}\right)$. Furthermore, if $L \neq L^{\prime}$ and if $T(L)$ and $T\left(L^{\prime}\right)$ are any subsets, of power $2^{\mathrm{N}_{0}}$ each, of $S(L)$ and $S\left(L^{\prime}\right)$ respectively, then $\overline{T(L)}$ and $\overline{T\left(L^{\prime}\right)}$ are incomparable order types $\left.{ }^{3}\right)$.

Proof. From Theorem 3.4 of [1], it follows that there exists a family of linear sets $\left\{Y_{\xi} \mid \xi<\theta\right\}$, each of which is both exact and has property $A$. Furthermore, for any two finite, linear sets $M$ and $N$, if $\xi \neq v$

1) A simply ordered set is exact if the only similarity transformation $f$ of $E$ into $E$ is the identity.

$\Rightarrow A$ linear set $E$, of power $2^{*_{0}}$, has property $A$ if no two disjoint subsets of $E$, of power $2^{* 0}$ each, are similar.

3) Let $C$ and $D$ be two simply ordered sets. $\vec{O}$ and $\bar{D}$ are said to be incomparable order types if there is no similarity transformation of $O$ into $D$ and no similarity transformation of $D$ into $C$. and $T_{\xi}$ and $T_{v}$ are subsets of power $2^{N_{0}}$ each of $Y_{\xi} \cup M$ and $Y_{v} \cup N$ respectively, then $\bar{T}_{\xi}$ and $\bar{T}_{v}$ are incomparable order types.

Now well order the set of lines in the plane into the sequence $\left\{L_{\xi}\right\}_{\xi<\theta}$. For each $\xi$ decompose $L_{\xi}$ into a family $\left\{B_{v}^{\xi} \mid v<\theta\right\}$ of pairwise disjoint sets, each $B_{v}^{\xi}$ being similar to $Y_{\xi}$. By Theorem 2.2 of [1] such decompositions are possible. Denote by $C_{0}$ the set $B_{0}^{0}$. Suppose that for each $\xi<\alpha<\theta$, the set $C_{\xi}$ has been defined so that $1^{0} C_{\xi}$ is one of the sets $B_{v}^{\xi}$, and $2^{0}$ the $C_{\xi}$ are pairwise disjoint. Since each $L_{\xi}$ is a line, for $\delta \neq \gamma$, the set $L_{\gamma} \cap L_{\delta}$ contains at most one element. The $B_{v}^{a}$ being pairwise disjoint for fixed $\alpha$, it follows that each set $C_{\xi}$ has a non-empty intersection with at most one set $B_{v}^{\alpha}$, say $B_{\mu(\xi)}^{\alpha}$. Since $\alpha<\theta$, there exists a set, call it $B_{v(\alpha)}^{\alpha}$, which has an empty intersection with each set $C_{\xi}, \xi<\alpha$. Define $C_{a}$ to be the set $B_{v(\alpha)}^{\alpha}$.

Well order the elements of the set $E^{2}-\bigcup_{\xi<\theta} O_{\xi}$ into a sequence $\left\{x_{\xi}\right\}_{\xi<\tau \leqslant \theta}$. Let $L_{\gamma(0)}$ be the first line which contains $x_{0}$. Now suppose that $\gamma(\xi)$ has been defined for $\xi<\delta<\tau$. Let $L_{\gamma(\delta)}$ be the first line not one of the lines $L_{\gamma(\xi)}, \xi<\delta$, which contains $x_{\delta} . L_{\gamma(\delta)}$ certainly exists since there are $2^{*_{0}}$ different lines containing $x_{\delta}$. If $v=\gamma(\xi)$ let $F_{v}=C_{n} \cup\left\{x_{\xi}\right\}$. If $v$ is not a $\gamma(\xi)$, let $F_{n}=C_{v}$. Clearly $E^{2}=\bigcup_{v<\theta} F_{n}$. Now each set $F_{v}$ is exact and has property $A$. From the selection of the sets $\Gamma_{\xi}$ it follows that if $T_{\xi}$ and $T_{v}$ are subsets, of power $2^{\mathrm{N}_{\theta}}$ each, of $F_{\xi}$ and $F_{v}$ respectively, then $\bar{T}_{\xi}$ and $\bar{T}_{v}$ are incomparable order types. Now decompose each set $F_{\xi}$ into disjoint pairs. This yields a decomposition of $E^{2}$ into disjoint pairs, since $E^{2}$ is the union of the pairwise disjoint sets $F_{\xi}$. There is no trouble in verifying that this decomposition satisfies the conclusions of Theorem 2.3 .

We conclude with the following questions:

Let $a<\lambda$ be a linear order type of power $2^{N_{6}}$. Does there exist a decomposition of the reals into disjoint pairs such that any set $S$ which is the union of pairs and which contains, as a subset, a set of order type $\alpha$, contains a subset of order type $\lambda$ ? If the answer is in the negative, then does there exist at least one such $\alpha$ ?

\section{References}

[1] Ginsburg, S., Further results on order types and decompositions of sets, Transactions of the American Mathematical Society 77 (1954), p. 122-150.

[2] Sierpiński, W., Un théorème de la théorie générale des ensembles et ses conséquences, Fundamenta Mathematicae 24 (1935), p. 8-11.

[3] - Sur deux problèmes de M. Ruziewicz concernant la décomposition de l'intervalle en paires de points, Fundamenta Mathematicae 24 (1935), p. 43-47.

Reçu par la Rédaction le 2S. 1. $19.5 t$ 